\title{
A GPS multipath mitigation technique using correlators with variable chip spacing
}

\author{
Jin Hyuk Lee ${ }^{1}$, Deok Won Lim², Jae Hee Noh¹, Gwang Hee Jo ${ }^{1}$, Chansik Park ${ }^{3}$, Jae Min Ahn ${ }^{4}$ and Sang Jeong Lee ${ }^{1, *}$ \\ ${ }^{1}$ Chungnam National University, Department of Electronics Engineering, 34134 Daejeon, Korea \\ ${ }^{2}$ Korea Aerospace Research Institute, Navigation R\&D Division, 34133 Daejeon, Korea \\ ${ }^{3}$ Chungbuk National University, Department of Electronics Engineering, 28644 Cheongju, Korea \\ ${ }^{4}$ Chungnam National University, Department of Radio and Information Communications Engineering, 34134 Daejeon, Korea
}

\begin{abstract}
Various methods have been studied to mitigate the influence of multipath signals, representative methods focused the correlator structure are the Narrow Correlator and the Multipath Elimination Technique (MET). It is known that the MET has better performance than Narrow Correlator but it requires more complexity. In this paper, we propose a technique that has similar performance to the MET and it uses only three correlators like the Narrow Correlator. This technique switches the chip spacing of the correlators for each Predetection Integration Time (PIT) and applies it to the MET. For the performance analysis, we implemented a software platform and compared the code tracking error of the proposed technique with that of the Narrow Correlator and the MET.
\end{abstract}

\section{Introduction}

In urban GNSS navigation applications, the presence of multipath is a dominant source of performance degradation in the pseudorange measurement and the positioning. In order to mitigate the effect of the multipath signal, various techniques have been studied. Among them, the early studies were focused on designing the antenna or correlator structure. A well-known algorithm is Narrow Correlator, which reduces the chip space between early and late correlator in order to cut down the impact of multipath on the Delay Lock Loop (DLL) [1]. However, this algorithm suffers from high sensitivity to noise due to the broad RF bandwidth, and it cannot mitigate short delay multipath. And the other well-known algorithm is the MET, which estimate the error by calculating an intersection point from the slope of a correlation function [2]. And it is known that the MET has better positioning performance than that of the Narrow Correlator, although it requires more complexity due to the increased number of correlators.

In this paper, we propose a multipath mitigation technique which has similar performance to the MET by using only three correlators. This technique switches the chip spacing between correlators every PIT. So, this technique can serve as five or more correlators with only three correlators. In this paper, the proposed technique obtains five correlation values and then applies those values to the MET algorithm. In order to evaluate the validity of the proposed technique, we compared the code tracking error with the Narrow Correlator and the MET through computer simulation. From that, it can be checked that the proposed technique shows better performance than the Narrow Correlator using the same number of correlators.
Also, it shows similar performance to the MET in a static environment, although it probably shows deteriorated performance due to a change in correlation value during PIT in a dynamic environment. Therefore, it can be expected that the proposed technique would be suitable for low receiver dynamics.

\section{Multipath signal}

GNSS receiver receive satellite navigation signals and calculates pseudoranges between the satellite and the receiver. After that, the receiver performs positioning using pseudoranges. However, satellite navigation signals incident on the receiver is not only a direct path signal but also signals reflected by obstacles. Signals reflected on the obstacles and incident on the receiver are called multipath signals. Figure 1 shows the direct path signal and the multipath signals incident on the receiver. The multipath signals have signal power attenuation, time delay, and phase delay compared to direct path signals. And these signals affect the code tracking loop in a receiver. When the direct path signal is received, the code discriminator on the code tracking loop is given in equation 1 [3].

$$
D(\tau)=R\left(\tau+\tau_{d}\right)-R\left(\tau-\tau_{d}\right)
$$

$D$ is the discriminator output. $R$ is the correlation function. $\tau_{d}$ is chip spacing between prompt correlator and early or late correlator. When a multipath signal is received, the code discriminator on the code tracking loop is given in equation 2 [3].

* Corresponding author: eesjl@cnu.ac.kr 


$$
D(\tau)=R\left(\tau+\tau_{d}\right)-R\left(\tau-\tau_{d}\right)+\alpha\left[R\left(\tau+\tau_{d}-\delta\right)-R\left(\tau-\tau_{d}-\delta\right)\right] \cos (\theta)(2)
$$

$\delta$ is relative chip delay between the direct path signal and the multipath signal. $\theta$ is relative phase between the direct path signal and the multipath signal. When the multipath signal is received, the discriminator output has an additional equation generated by the multiappath signal. The additional equation is a bias error in the code tracking loop. Therefore, in the pseudorange calculation, the receiver generates a bias error and increases the navigation error due to the influence of the multipath signal.

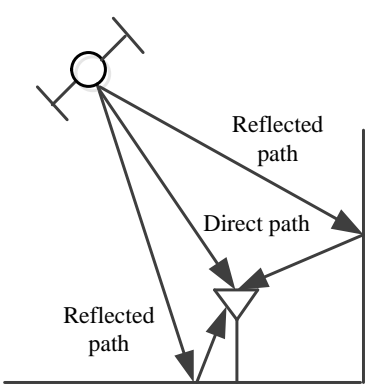

Fig. 1. Direct path and multipath signals.

\section{Multipath elimination technique}

\subsection{Narrow Correlator}

The Narrow Correlator has the simple structure with only three correlators. A typical receiver sets the chip space between the early correlator and the late correlator to 1.0 chip for signal tracking. The Narrow Correlator, unlike typical receivers, sets the chip space to less than 0.2 chip. By reducing the correlator chip space in this way, the pseudorange error caused by the multipath signal can be reduced. Figure 2 shows that the code phase error is reduced by reducing correlator spacing.

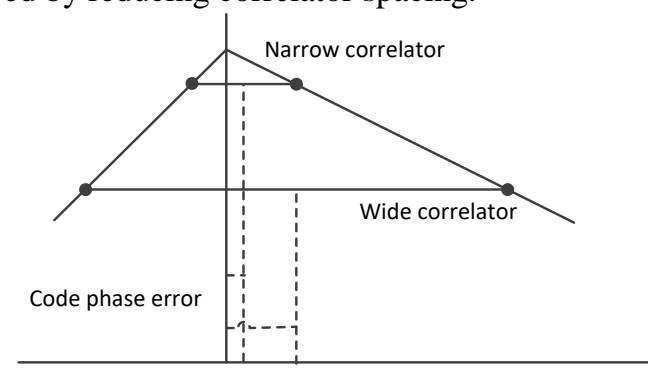

Fig. 2. Code phase error for the wide correlator and the narrow correlator.

\subsection{Multipath Elimination Technique}

The MET computes code phase error using slopes of the correlation function with five correlators. The arrangement of the correlator is two early correlators, one prompt correlator, and two late correlators. The two early correlation values are connected to form a line, and the two rate correlation values are also connected to form a line. Then calculate the $\mathrm{x}$-coordinate of the intersection of the two lines. This $x$ value is the code tracking error estimated by the MET and is shown in Equation 3. Figure 3 shows that concept of the MET.

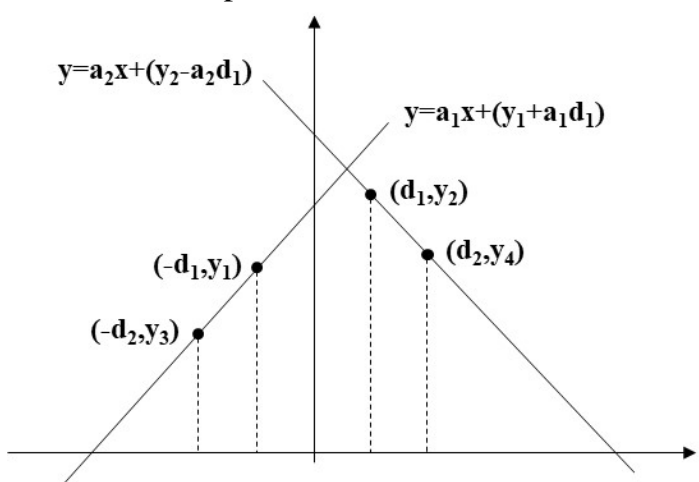

Fig. 3. Multipath Elimination Technique.

$$
T=\frac{y_{1}-y_{2}+d / 2\left(a_{1}+a_{2}\right)}{a_{1}-a_{2}}
$$

$y_{1}$ and $y_{2}$ are the correlation values. $d$ is the chip spacing of early correlator and late correlator. $a_{1}$ is the slope of the early correlators. $a_{2}$ is the slope of the late correlators. $T$ is the code tracking error.

\section{Proposed Technique}

The proposed technique uses three correlators like the Narrow Correlator. This technique switches the chip space between correlators every PIT and stores correlation values. In detail, this technique uses two early correlators for the first PIT and two late correlators for the next PIT as shown if Figure 4. And then this technique applies these four stored correlation values to the MET.

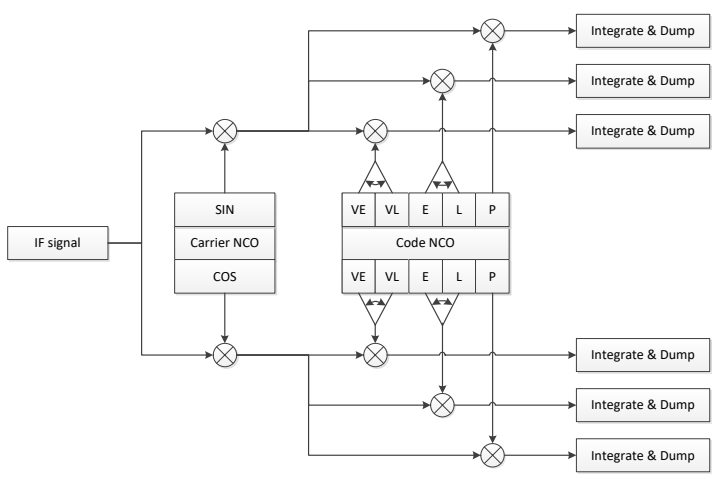

Fig. 4. Correlator structure of proposed technique.

\section{Simulation}

\subsection{Simulation platform}

By using the software based simulation platform, performance of multipath error mitigation techniques have been compared. The simulation platform is shown in Figure 5. The simulation platform consists of a signal generator and a signal receiver. The signal generator sets the scenarios and parameters, and then generates GPS signal and multipath signal. The signal receiver processes the IF signal generated by the signal generator and 
acquires and tracks the signal to find the code phase. At the signal acquisition part, the approximate signal code chip and Doppler frequency are found. At the signal tracking part, Frequency Lock Loop (FLL), Phase Lock Loop (PLL) and Delay Lock Loop (DLL) are equipped, and the multipath error mitigation technique is implemented in DLL. Code phase error can be obtained by subtracting the code phase from the signal receiver and the signal generator.

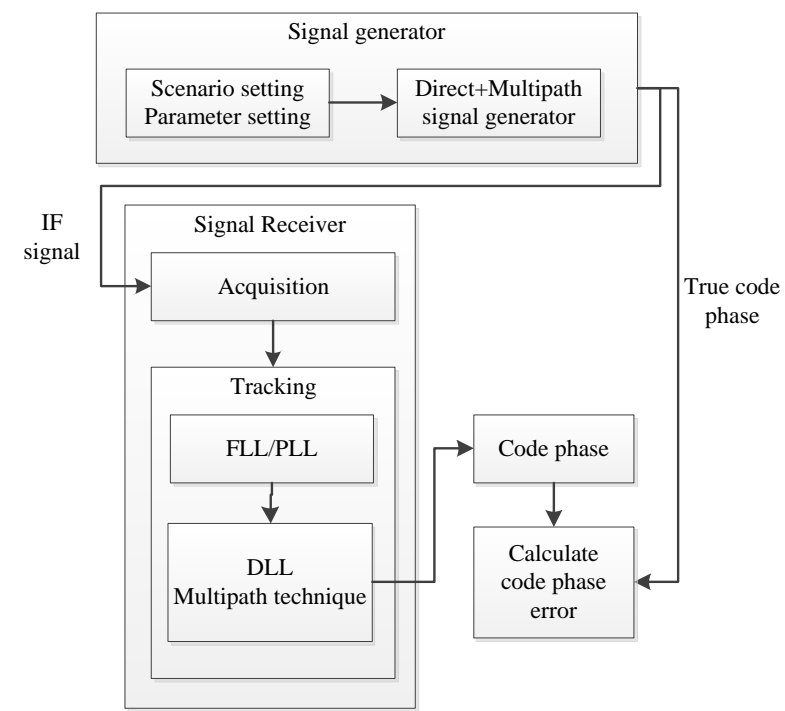

Fig. 5. The performance analysis platform of multipath mitigation techniques.

\subsection{Simulation setup}

In order to verify the effectiveness of the proposed technique, we implemented the Narrow Correlator, MET, and proposed technique, and then compared code tracking performance for those techniques. The dynamics of the receiver is static. The relative signal power, relative phase and relative chip delay of the direct path signal and the multipath signal are specified in the table 1 .

Table 1. Simulation Setup.

\begin{tabular}{|c|c|}
\hline Parameter & Setting \\
\hline Relative amplitude & 0.5 \\
\hline Relative phase & 0,180 [degree] \\
\hline Relative chip delay & $0-1.1$ [chip] \\
\hline Receiver dynamics & Static \\
\hline Correlator chip space & 0.05 [chip] \\
\hline
\end{tabular}

\subsection{Results}

Figure 6 shows the code phase errors according to the multipath delay of the tree techniques. From that, it can be checked that the proposed technique has better performance than the Narrow Correlator and has similar performance to the MET.

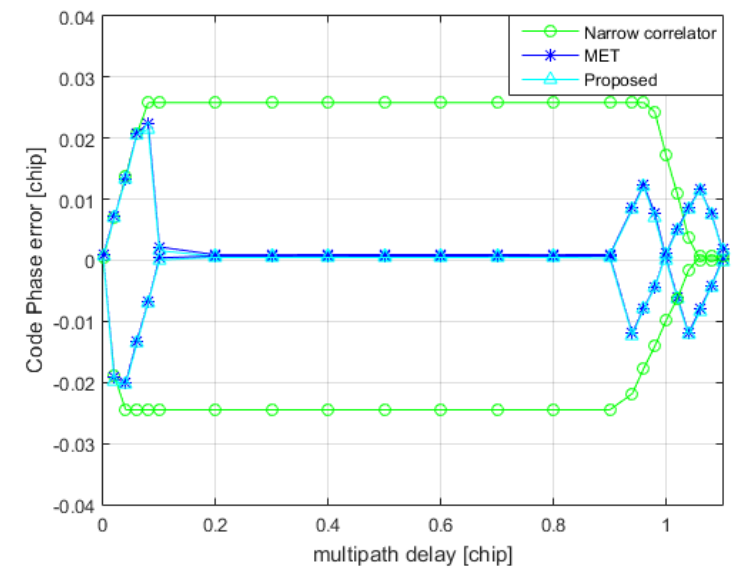

Fig. 6. Multipath error envelope.

\section{Conclusions}

In this paper, we propose a technique that has similar performance to the MET by using only three correlators like the Narrow Correlator. And we designed software platform to confirm the validity of the proposed technique. Based on the platform, we compared the code tracking error of the proposed technique with that of the Narrow Correlator and the MET. From the results, it can be confirmed that the proposed technique has better performance than the Narrow Correlator and has similar one to the MET by using only three correlators.

\section{Acknowledgement}

This work has been supported partly by the National GNSS Research Center Program of Defense Acquisition Program Administration and the Agency for Defense Development and also supported by the Research Fund of Chungnam National University

\section{References}

1. A. J. Van Dierendonck, P. Fenton, T. Ford, Theory and Performance of Narrow Correlator Spacing in a GPS Receiver, ION 39, 3, pp.265-283 (1992)

2. B. Townsend, P. Fenton, A Practical Approach to the Reduction of Pseudorange Multipath Errors in A L1 GPS Receiver, Proceedings of ION GPS-94, pp. 143148 (1994)

3. BW. Parkinson, P. Enge, P. Axelrad, JJ. Spilker Jr, Global Positioning System: Theory and Applications Volume I (American Institute of Aeronautics and astronautics, DC, Washington, 1996) 